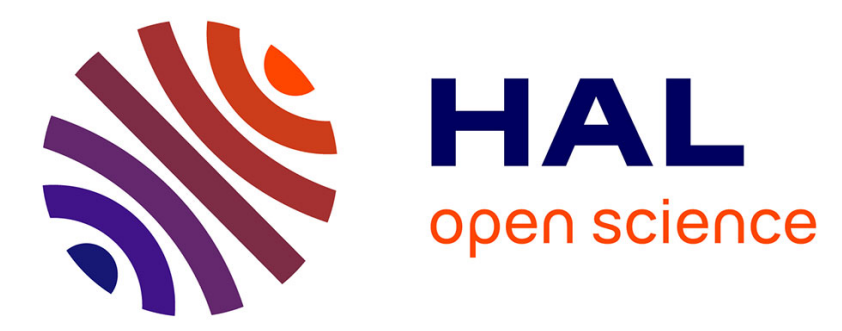

\title{
Shear wave elastography of lumbar annulus fibrosus in adolescent idiopathic scoliosis before and after surgical intervention
}

Claudio Vergari, Lucas Chanteux, Raphaël Pietton, Tristan Langlais, Raphaël Vialle, Wafa Skalli

\section{To cite this version:}

Claudio Vergari, Lucas Chanteux, Raphaël Pietton, Tristan Langlais, Raphaël Vialle, et al.. Shear wave elastography of lumbar annulus fibrosus in adolescent idiopathic scoliosis before and after surgical intervention. European Radiology, 2020, 30 (4), pp.1980-1985. 10.1007/s00330-019-06563-4 . hal02531525

\section{HAL Id: hal-02531525 \\ https://hal.science/hal-02531525}

Submitted on 3 Apr 2020

HAL is a multi-disciplinary open access archive for the deposit and dissemination of scientific research documents, whether they are published or not. The documents may come from teaching and research institutions in France or abroad, or from public or private research centers.
L'archive ouverte pluridisciplinaire HAL, est destinée au dépôt et à la diffusion de documents scientifiques de niveau recherche, publiés ou non, émanant des établissements d'enseignement et de recherche français ou étrangers, des laboratoires publics ou privés. 


\title{
Shear wave elastography of lumbar annulus fibrosus in adolescent idiopathic scoliosis before and after surgical intervention
}

\author{
Claudio Vergari $^{1}(\mathbb{D}) \cdot$ Lucas Chanteux $^{1,2} \cdot$ Raphael Pietton $^{2} \cdot$ Tristan Langlais $^{1,2} \cdot$ Raphael Vialle $^{2} \cdot$ Wafa Skalli $^{1}$
}

\begin{abstract}
Objectives To determine lumbar intervertebral disc properties with shear wave elastography in adolescent idiopathic scoliotic (AIS) patients before and after surgery, and compare them with asymptomatic controls.

Methods Twenty-five severe AIS patients with an indication for fusion surgery (15 \pm 1.5 years old, the Cobb angle ranging between 40 and $93^{\circ}$ ) and fifty-nine asymptomatic adolescents ( $13 \pm 2$ years old) were included prospectively. Shear wave speed (SWS) was measured in the annulus fibrosus of L3-L4, L4-L5, and L5-S1 discs of each subject. In AIS patients, measurements took place before surgery, and 3 months $(N=13)$ or 1 year after $(N=12)$.

Results No difference was observed between disc levels in any group. When pooling disc levels, SWS was significantly higher in preop AIS patients $(4.0 \pm 0.5 \mathrm{~m} / \mathrm{s})$ than in asymptomatic subjects $(3.1 \pm 0.5 \mathrm{~m} / \mathrm{s}, p<0.0001)$. SWS decreased 3 months postop $(3.5 \pm 0.3 \mathrm{~m} / \mathrm{s})$, and it decreased further towards normal values 1 year after $(3.3 \pm 0.4 \mathrm{~m} / \mathrm{s})$. SWS in preop AIS patients tended to decrease with the Cobb angle (Spearman's rho $=-0.4, p=0.05$ ).

Conclusion Shear wave elastography measurements showed that discs in AIS patients were altered relative to asymptomatic ones, and this alteration tended to normalize 1 year post fusion surgery. Further studies should aim at determining if bracing of mild scoliosis has an effect on disc properties.
\end{abstract}

Key Points

- Shear wave elastography shows alteration of annulus fibrosus in adolescent idiopathic scoliosis.

- Disc elastography tends to normalize 1 year after surgery.

Keywords Elasticity imaging techniques $\cdot$ Spine $\cdot$ Intervertebral disc $\cdot$ Arthrodesis $\cdot$ Orthopedics

\section{Abbreviations}

AIS Adolescent idiopathic scoliosis

BMI Body mass index

SWS Shear wave speed

\section{Introduction}

Adolescent idiopathic scoliosis (AIS) is the main symptom of a multifactorial pathology, the etiopathogenesis of which is

Claudio Vergari

c.vergari@gmail.com

1 LBM/Institut de Biomécanique Humaine Georges Charpak, Arts et Métiers ParisTech, 151 bd de l'Hôpital, 75013 Paris, France

2 Department of Paediatric Orthopaedics, Armand Trousseau Hospital, Université Pierre et Marie Curie-Paris 6, 75571 Paris, France still unknown. According to the scoliosis research society, scoliosis is mainly characterized by a Cobb angle higher than $10^{\circ}$ developing after 10 years of age, and it presents a high risk of progression during the growth spurt.

Beyond the Cobb angle, a parameter measurable in the patient's coronal plane, other characteristics are specific to AIS, such as a rotation of the vertebrae in the axial plane and an increased stiffness of the spine [1]. Both these aspects could be related to alterations of the intervertebral disc. Indeed, the disc likely plays a role in the scoliotic deformity: recent in vivo study showed that scoliotic discs present a stiffer annulus fibrosus than asymptomatic adolescents [2]. Those measurements were performed with shear wave elastography, a non-invasive and ultrasound-based technique that can quantitatively evaluate soft tissue mechanical properties [3].

When conservative treatment of the deformity fails, and progression leads to severe scoliosis (Cobb angle $>50^{\circ}$ ), surgery can be necessary to avoid respiratory impairment and to 
improve the patient's quality of life. The most common approach is to fuse the scoliotic curve to avoid further progression of the deformity, while leaving some spinal segments free to allow the trunk some mobility [4]. Thus, while the discs within the instrumented segment almost completely lose their mobility, those outside the fusion can undergo larger strains than usual to compensate and allow the patient to perform every-day tasks. Therefore, assessing disc status may be informative for the surgeon when choosing which vertebral levels should be included in the instrumentation.

Magnetic resonance imaging is usually used to assess intervertebral disc. For instance, it has been shown that discs below the fusion can tend to recover hydration 2 years postoperatively [5] but also, in more than half the cases, undergo accelerated disc degeneration [6]. Still, magnetic resonance imaging remains of difficult access in clinical routine, and it does not provide information on disc mechanical properties in vivo.

The aim of this study was to determine if any change in annulus fibrosus mechanical properties could be detected after fusion surgery in AIS patients with shear wave elastography.

\section{Material and methods}

\section{Subjects}

Data were collected prospectively from the Trousseau University children's hospital within follow-up clinical routine, between November 2016 and June 2018. Twenty-five severe AIS patients were included prospectively in this study (18 girls and 7 boys, $15 \pm 1.5$ years old, ranging between 13 and 17 years. Cobb angle $57 \pm 14^{\circ}$, ranging between 40 and 93 $\left.{ }^{\circ}\right)$. Fifteen AIS patients had thoracic curves, nine had thoracolumbar curves, and one a lumbar curve; according to Lenke's classification [7], 15 patients were type A, 6 were type B, and 4 type C. All patients had indications for spine fusion surgery. Patients presenting transitional anomalies, non-idiopathic scoliosis, or antecedent connective tissue pathology or spinal surgery were excluded.

Fifty-nine asymptomatic subjects were also included as control group ( 36 girls and 23 boys, $13 \pm 2$ years old, ranging between 10 and 16 years).

Height and weight were measured for all subjects, and body mass index (BMI) was calculated. All subjects signed informed consent, as approved by the ethical committee (C.P.P Île de France IV \#14 409).

\section{Shear wave elastography}

Shear wave speed (SWS) was measured with an Aixplorer (SuperSonic Imagine) and a superlinear SL 10-2 MHz probe with a previously described protocol [8]. Briefly, patients were in supine position and the probe was placed on the abdomen to image the spine with an anterior approach. Constant pressure was applied with the probe, which allowed displacing the intestinal contents, thus improving ultrasound penetration. The aortic bifurcation was localized to determine the L4 vertebral level, and the probe was subsequently moved up or down to measure SWS at three discs levels: L3-L4, L4-L5, and L5-S1. The orientation of the probe was adjusted to be in the plane of the disc; the orientation was deemed acceptable when the annulus lamellae were visible, and a reliable shear wave image was obtained (i.e., an image with little to no localized saturation of the color chart or absent signal, and a constant color chart in time).

\section{Protocol}

Measurements were performed preoperatively for all AIS patients, and they were repeated 3 months after surgery in 13 patients and 1 year after surgery in the remaining 12 patients. No patients were lost at follow-up, and surgery was performed blinded to SWS results. For each measurement, three 10-s clips of elastography were acquired, corresponding to about 30 elastographic images. Images were processed in custom software developed in MATLAB (The MathWorks) to select the annulus fibrosus in each image and calculate the corresponding average SWS. Images with missing signal or saturation were discarded. Then, the SWSs measured in the remaining images were averaged to obtain a single SWS value for each measurement [9]. All measurements and processing were performed by two trained operators, while a third blinded operator verified the processing.

\section{Statistics}

Non-parametric statistical tests were used because not all variables were normally distributed (Lilliefors normality test). Kruskal-Wallis tests were used to compare the three vertebral levels and the four groups (asymptomatic, preop, 3 months postop, 1 year postop). Correlations were analyzed with Spearman's rank test. Significance was $p<0.05$. Analyses were performed in MATLAB 2016b (The MathWorks)

\section{Results}

Figure 1 shows examples of echographic and elastographic images at all disc levels considered. Figure 2 reports SWS in annulus fibrosus at the three disc levels for the four groups (asymptomatic, preop AIS, 3 months postop, and 1 year postop). Within each group, no difference was observed between disc levels $(p>0.05)$, so Fig. 2 also reports values for pooled levels; when pooling all levels, SWS was $3.1 \pm 0.5 \mathrm{~m} / \mathrm{s}$ in asymptomatic subjects and significantly higher in preop AIS, at $4.0 \pm 0.5 \mathrm{~m} / \mathrm{s}$ $(p<0.0001)$. Three months postop, SWS decreased to $3.5 \pm 0.3$, 


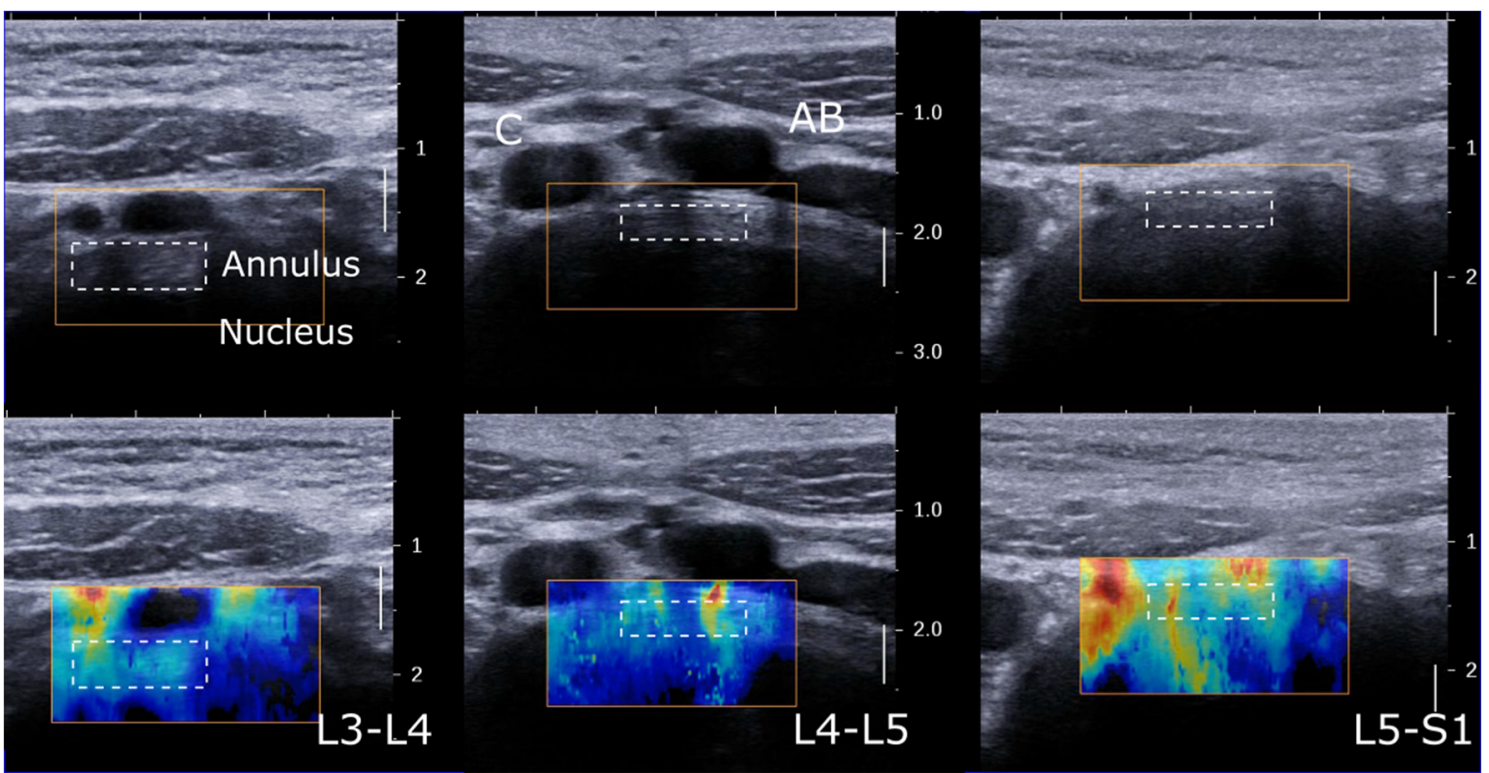

Fig. 1 Example of annulus fibrosus echography and shear wave elastography at L3-L4, L4-L5, and L5-S1 disc levels. Nucleus appears hypoechoic. Aortic bifurcation $(\mathrm{AB})$ and inferior vena cava $(\mathrm{C})$ are visible

and it further decreased to $3.3 \pm 0.4 \mathrm{~m} / \mathrm{s}$ after 1 year. Preop SWS was significantly higher than in asymptomatic patients at all disc levels $(p<0.0001)$. The SWS decrease after 3 months postop was not significant, but it became significant after 1 year at all disc levels $(p<0.0001)$, when SWS was similar to that of asymptomatic controls $(p>0.05)$.

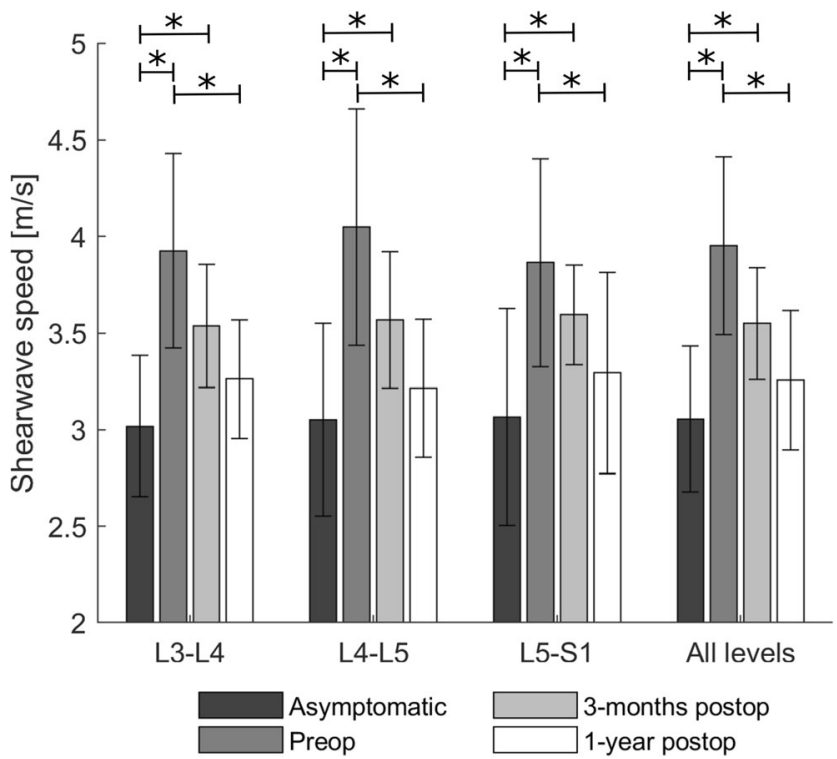

Fig. 2 Shear wave speed (SWS) of the annulus fibrosus at L3-L4, L4-L5, and L5-S1 disc levels (average \pm standard deviation), and all levels pooled. Comparison between asymptomatic subjects, preoperative adolescent idiopathic scoliosis patients, and 3-month and 1-year postsurgery patients. Preop measurements were significantly higher than in asymptomatic subjects and 1 year postop ( $p<0.001$, Kruskal-Wallis test), while 1 year postop was significantly lower than preop in all disc levels $(p<0.001)$ at the L4-L5 level. Dashed line rectangles show placement of region of interest for shear wave speed measurement

Three months postop, only 4 patients had slightly increased their SWS by $0.1 \mathrm{~m} / \mathrm{s}$ in average, while the rest decreased by $0.7 \mathrm{~m} / \mathrm{s}$. One year postop, only one patient appeared to increase her SWS by $0.2 \mathrm{~m} / \mathrm{s}$, while the rest decreased by $0.8 \mathrm{~m} / \mathrm{s}$. A normality corridor can be defined based on the asymptomatic population as the 5-95\% quantiles, which results as the range 2.3 and $3.8 \mathrm{~m} / \mathrm{s}$. Before surgery, $44 \%$ of the measured discs were above this corridor (and none was below). Three months after surgery, $26 \%$ were above the corridor, and this percentage decreased to $10 \% 1$ year postop.

Thoracic (SWS $=3.8 \pm 0.4$ ) and thoracolumbar AIS patients (SWS $=3.9 \pm 0.4)$ showed similar SWS $(p>0.05)$; the only patient with a lumbar curve showed an average SWS of $5.1 \mathrm{~m} / \mathrm{s}$, which was the highest value observed. Patients which were classified as Lenke type $\mathrm{C}(n=4$, corresponding to 12 discs) showed significantly higher SWS (4.4 \pm $0.7 \mathrm{~m} / \mathrm{s})$ than B-type $(3.7 \pm 0.6 \mathrm{~m} / \mathrm{s}, p=0.001)$ and A-type patients $(3.8 \pm 0.4 \mathrm{~m} / \mathrm{s}, p=0.04)$. Still, all three types had significantly higher SWS than asymptomatic subjects $(p<0.0001)$.

Asymptomatic patients showed no correlation between SWS and age, height, weight, or BMI. AIS patients only showed a weak positive correlation preop with age (rho = $0.3, p=0.007$ ), but not postop. A weak correlation was also observed between SWS (all disc levels pooled) and Cobb angle (Fig. 3, Spearman's rho $=-0.4, p=0.05$ ). When comparing SWS in AIS patients with the $95 \%$ normality corridor of asymptomatic subjects (shaded area in Fig. 3), it can be noticed that all patients are in the top half of the normality corridor and that $52 \%$ of them (13 patients) are above it. 


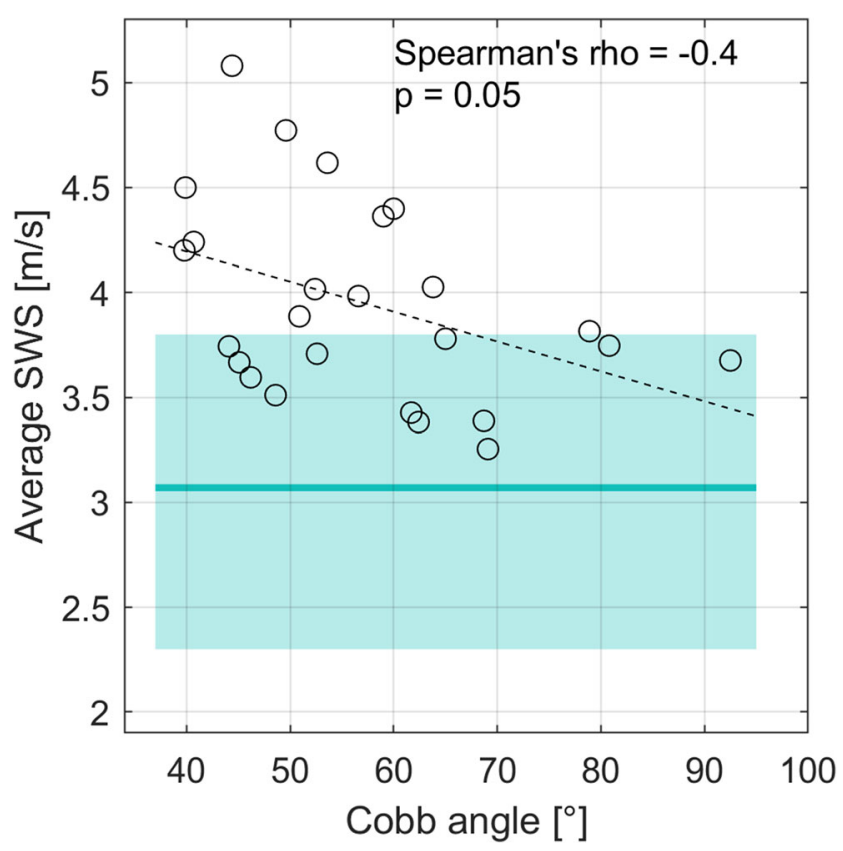

Fig. 3 Relationship between Cobb angle and the average shear wave speed (SWS) of disc levels between L3-L4 and L5-S1 (Spearman's rho $=-0.4, p=0.05$ ). The dashed line is a linear regression between variables, while the horizontal line and the shaded area represent the average and $95 \%$ normality corridor of asymptomatic subjects, respectively

\section{Discussion}

In this study, scoliotic intervertebral disc was assessed preoperatively and postoperatively, for the first time, by shear wave elastography. A higher SWS is associated to a stiffer tissue; indeed, SWS can be directly related to the tissue's shear modulus $(\mu)$ and Young's modulus $(E)$ by the following relationship: $S W S=\sqrt{\mu / \rho} \approx \sqrt{3 E / \rho}$, where $\rho$ is the tissue mass density. This relationship allows calculating the shear modulus from SWS, but it is only valid under several assumptions, such as tissue homogeneity, non-dispersivity, and non-compressibility $[10,11]$. Since biological tissues rarely satisfy these assumptions, only SWS is reported in this work. Results confirm previous measurements reporting that scoliotic annulus fibrosus shows higher SWS, but they also show that the annulus start normalizing 3 months after surgery and can attain normal values 1 year later.

Surgery aims at straightening and fusing the scoliotic curve, but there is still no consensus on the choice of which vertebrae should be included in the fusion. On the one hand, long fusion will better stabilize the spine while on the other hand, shorter instrumentation improves trunk mobility [12]. In particular, the choice of the last instrumented vertebra is important to avoid adjacent disc disease or degeneration $[5,13]$, but again, no universal rule exists.
Shear wave elastography is a rapid and non-invasive measurement which can be performed at bedside; potentially, it could play a key role in helping the surgeon choosing the last instrumented level. Moreover, it could be potentially employed to detect early a degeneration of the adjacent disc postoperatively. Future work should aim at including larger cohorts to analyze more in detail those discs adjacent to the lower junctional vertebra (defining the lower end of the scoliotic curve), to the stable vertebra (the most closely bisected by a vertically directed central sacral line) and the neutral disc (vertebra with the least axial rotation) [14]. These vertebrae are critical in the assessment of spinal deformities, and therefore, their adjacent discs could present specific characteristics. Future work could also assess the feasibility of standing disc elastography, which could be informative on disc changes when loaded, while intraoperative elastography could provide a strong validation of the measurement, which would not be affected by the surrounding soft tissues, although anterior approach is rare in scoliosis surgery.

The main limitation of the present study is that the cohort was not large enough to perform a sub-analysis on disc levels adjacent to instrumentation: only 5 patients were fused down to a vertebra below L3; thus, only 5 discs adjacent to the instrumentation were measured, and only 4 patients (16 discs) were type C. Nevertheless, results suggest that deformity in the lumbar region further affects the intervertebral discs, although lumbar discs appear stiffer than asymptomatic ones even when the deformity if limited to the thoracic region (A-type patients and thoracic curves).

In the proposed protocol, vertebral level was determined relative to the aortic bifurcation; however, this landmark is at the L4 vertebral level only in $64 \%$ of the population [15]. Other means exist for robust determination of the level at which the elastography is performed, especially in preoperative patients. First of all, patients usually undergo magnetic resonance imaging, which allows locating the aortic bifurcation relative to vertebral level. Second, standing radiography is routinely performed, which shows the relationship between the iliac crests, which can be easily located during ultrasonography, and the spinal levels. Finally, it is possible to localize with ultrasound the lowest visible disc and move upwards to find the following discs. Therefore, with a combination of these methods, a robust identification of the imaged disc level is possible.

A limit intrinsic to the technique is that measurements are performed with an anterior approach, which does not allow accessing the thoracic discs. Thoracic curves still showed high SWS in lumbar discs, indicating that disc alteration reaches outside the curve. Nevertheless, the only lumbar patient included showed the highest SWS measured in this study; while no conclusion can be drawn on a single measurement, it does not appear too speculative to hypothesize that those discs 
within or adjacent to the curve can more affected than others by altered mechanical properties.

Finally, postprocessing is performed in MATLAB, and the processing of a single patient (i.e., three films times three discs) takes about $10 \mathrm{~min}$. This might not yet be compatible with clinical routine, but more sophisticated techniques could be employed to speed up processing time, for instance based on machine learning for automatic ROI selection and tracking. Acquiring a large number of elastographic images allows averaging SWS on a relatively long period (about $30 \mathrm{~s}$ ), thus smoothing potential periodical variations due to respiration or arterial pulsation.

Reproducibility of shear wave elastography in lumbar annulus fibrosus is $0.5 \mathrm{~m} / \mathrm{s}$, in terms of $95 \%$ confidence interval [16], which is smaller than the difference between asymptomatic and AIS subjects measured in this study $(3.1 \pm 0.4 \mathrm{~m} / \mathrm{s}$ versus $4.0 \pm 0.5$, respectively), and smaller than the postoperatively decrease of SWS $(-0.7 \mathrm{~m} / \mathrm{s}$ at 1 year). This confirms that the difference between the two populations is clinically relevant. Although most discs normalized their SWS 1 year postop, they did so at different rates; future studies should aim at establishing a relationship between preoperative disc SWS, its short-term change postoperatively, and the longer term clinical outcome.

SWS tended to decrease with higher Cobb angles. This is surprising because AIS patients have higher SWS than asymptomatic controls, so an increase of SWS with curve severity could be expected. However, more severe patients would have been treated by bracing more aggressively and for longer time than less severe patients. It is possible that bracing influenced disc hydration, and therefore its mechanical properties as estimated by shear wave elastography. This hypothesis could be tested on a group of mild scoliotic patients before and during brace treatment.

In conclusion, this study confirmed previous shear wave elastography assessment of scoliotic discs, reporting that annulus fibrosus is stiffer in AIS. Moreover, data shows that annulus started normalizing 3 months after surgery and had attained normal SWS 1 year postop. Further work should be based on larger cohorts allowing for more in-depth analysis on key disc levels (relative to the scoliotic curve). However, these results are promising, since they show that shear wave elastography can assess alterations of the scoliotic annulus fibrosus and their change in time after surgery.

Funding information The authors are grateful to the DHU MAMUTH and ParisTech BiomecAM chair program on subject-specific musculoskeletal modeling (with the support of ParisTech and Yves Cotrel Foundations, Société Générale, Covea and Proteor).

\section{Compliance with ethical standards}

Guarantor The scientific guarantor of this publication is Prof. Wafa Skalli.
Conflict of interest The authors of this manuscript declare no relationships with any companies, whose products or services may be related to the subject matter of the article.

Statistics and biometry Several of the authors have significant statistical expertise.

Informed consent Written informed consent was obtained from all subjects and patients in this study.

Ethical approval Institutional Review Board approval was obtained (C.P.P Île de France IV \#14 409).

\author{
Methodology \\ - prospective \\ - experimental study \\ - performed at one institution
}

\section{References}

1. Deviren V, Berven S, Kleinstueck F, Antinnes J, Smith JA, Hu SS (2002) Predictors of flexibility and pain patterns in thoracolumbar and lumbar idiopathic scoliosis. Spine (Phila Pa 1976) 27:23462349. https://doi.org/10.1097/01.BRS.0000030300.45392.BF

2. Langlais T, Vergari C, Pietton R, Dubousset J, Skalli W, Vialle R (2018) Shear wave elastography can evaluate annulus fibrosus alteration in adolescent scoliosis. Eur Radiol 28:2830-2837

3. Gennisson JL, Deffieux T, Fink M, Tanter M (2013) Ultrasound elastography: principles and techniques. Diagn Interv Imaging 94: 487-495. https://doi.org/10.1016/j.diii.2013.01.022

4. Marks M, Newton PO, Petcharaporn M et al (2012) Postoperative segmental motion of the unfused spine distal to the fusion in 100 patients with adolescent idiopathic scoliosis. Spine (Phila Pa 1976) 37:826-832

5. Abelin-Genevois K, Estivalezes E, Briot J, Sévely A, Sales de Gauzy J, Swider P (2015) Spino-pelvic alignment influences disc hydration properties after AIS surgery: a prospective MRI-based study. Eur Spine J 24:1183-1190. https://doi.org/10.1007/s00586015-3875-4

6. Green DW, Lawhorne TW 3rd, Widmann RF et al (2011) Longterm magnetic resonance imaging follow-up demonstrates minimal transitional level lumbar disc degeneration after posterior spine fusion for adolescent idiopathic scoliosis. Spine (Phila Pa 1976) 36:19481954. https://doi.org/10.1097/BRS.0b013e3181fflea9

7. Lenke LG, Betz RR, Harms J et al (2001) Adolescent idiopathic scoliosis: a new classification to determine extent of spinal arthrodesis. J Bone Joint Surg Am 83-A:1169-1181

8. Vergari C, Rouch P, Dubois G et al (2014) Non-invasive biomechanical characterization of intervertebral discs by shear wave ultrasound elastography: a feasibility study. Eur Radiol 24:3210 3216. https://doi.org/10.1007/s00330-014-3382-8

9. Vergari C, Rouch P, Dubois G et al (2014) Intervertebral disc characterization by shear wave elastography: an in vitro preliminary study. Proc Inst Mech Eng H 228:607-615. https://doi.org/10. 1177/0954411914540279

10. Deffieux T, Gennisson JL, Bercoff J, Tanter M (2011) On the effects of reflected waves in transient shear wave elastography. IEEE Trans Ultrason Ferroelectr Freq Control 58:2032-2035. https://oi.org/ 10.1109/TUFFC.2011.2052

11. Bercoff J, Tanter M, Fink M (2004) Supersonic shear imaging: a new technique for soft tissue elasticity mapping. IEEE Trans Ultrason Ferroelectr Freq Control 51:396-409. https://doi.org/10. 1109/TUFFC.2004.1295425 
12. Fischer CR, Kim Y (2011) Selective fusion for adolescent idiopathic scoliosis: a review of current operative strategy. Eur Spine J 20: 1048-1057. https://doi.org/10.1007/s00586-011-1730-9

13. Ilharreborde B, Morel E, Mazda K, Dekutoski MB (2009) Adjacent segment disease after instrumented fusion for idiopathic scoliosis: review of current trends and controversies. J Spinal Disord Tech 22: 530-529

14. O’Brien MF, Kulklo TR, Blanke KM, Lenke LG (2008) Radiographic measurement manual. Spinal deformity study group (SDSG). Medtronic Sofamor Danek USA, Inc
15. Deswal A, Tamang BK, Bala A (2014) Study of aortic- common iliac bifurcation and its clinical significance. J Clin Diagn Res 8: AC06-AC08. https://doi.org/10.7860/JCDR/2014/8767.4559

16. Vergari C, Dubois G, Vialle R et al (2016) Lumbar annulus fibrosus biomechanical characterization in healthy children by ultrasound shear wave elastography. Eur Radiol 26:1213-1217. https://doi. org/10.1007/s00330-015-3911-0 